diversity of both providers and users of health services, every effort should now be made to ensure that assessment is done by a professional who has an understanding of the patient's culture. The authors state that systems are not required to be colour-blind, as this is not helpful. What is required is an acknowledgement of the disadvantages that people from ethnic minorities face and the implementation of measures to address these: denial is not the way forward.

The authors point out the fault lines in the assessment of dangerousness by psychiatrists and the tendency to be more cautious now, thereby leading to overprediction of dangerousness. They argue that this results in greater overprediction in Black people, which in turn is partly responsible for their overrepresentation in secure settings. The suggestion that psychiatry should be explicit about the limitations of making judgements regarding individual dangerousness is welcome, but this should not preclude offering an informed view on the available information and also on the interplay between mental disorder and dangerousness, where indicated. A move away from illness analysis to 'real-life analysis' is advocated, but this view needs more elucidation.

The authors suggest increasing resources to institutions run by Black mental health professionals that aim to provide alternatives to psychiatric hospitals. However, such facilities can at best serve as adjuncts, because many patients, whatever their racial background, need the type of care provided in mainstream psychiatric hospitals, and it is the provision of care in these hospitals that should reflect the diverse cultural and ethnic needs of their users.

The authors argue that appointing more professionals from ethnic minorities in the mental health services does not generally lead to improvement in service provision, because of constraints on those individuals. They note that people previously identified as radical become conservative on being appointed to higher positions. Although therefore questioning the drive to recruit increasing numbers of people from ethnic minorities, the authors also note the relatively small number of Black people in senior positions in various parts of the criminal justice system. Increasing these numbers should strengthen their influence on the changes required for a true multiethnic society. Perhaps a way of assuaging the main concern of the authors is for Black people appointed to senior positions to be made to sign a 'Radicality Act' just like the Official Secrets Act, to ensure that they remain radical after their appointment.

Deji Oyebode Clinical Director of Forensic Mental Health Services, Springfield University Hospital, Shaftesbury Clinic, Glenburnie Road, London SWI7 7DJ, UK

\section{Atypical Antipsychotics}

Edited by B. A. Ellenbroek \& A. R. Cools. Basel: Birkhäuser. 2000. 236 pp.

ISBN $376435948 \times$

This is the best, most concise and comprehensive account of all aspects of atypical antipsychotics that I have seen.

Although atypical antipsychotics have been established as treatments for schizophrenia for nearly 10 years, and the science underpinning their discovery is well described and goes back 30 years, atypicals are the subject of a controversy that frustrates their routine use, particularly in the UK. Consequently, only a minority of patients are on these superior drugs.

Part of the problem is that many different disciplines have a say in this field, from molecular biologists and behavioural psychologists through to clinicians, economists and politicians. Only the most conservative opinions and practices seem to prevail, even in the face of overwhelming evidence for the efficacy of the atypicals.

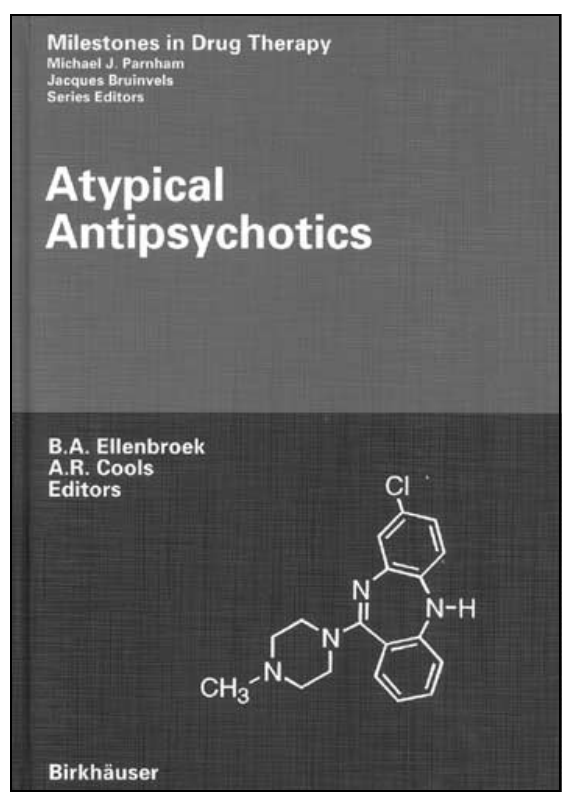

Such is the concern that there is even a review of these agents by the National Institute for Clinical Excellence.

The strength of this volume is that it brings together all of these areas under one cover. There are comprehensive chapters about the drug discovery process, receptor nomenclature and pharmacology, animal models of schizophrenia and imaging. There are also short reviews of each drug that include their clinical characteristics and pharmacological and neurochemical profiles. This is a valuable one-stop source of reference in this field.

The book is not perfect: a multi-review format usually has some overlap as well as variability in quality. Although this collection is the best of its type, some of the reviews are a little one-sided and thinly referenced. However, the majority of chapters are models of clarity and comprehensiveness. I would particularly congratulate Meltzer and Stahl on their contributions.

This is a vital addition to the shelf of every psychopharmacologist and a must for every medical school and research institute library.

\section{Robert Kerwin Professor of Clinical}

Neuropharmacology, Division of Psychological Medicine, Institute of Psychiatry, De Crespigny Park, Denmark Hill, London SE5 8AF, UK

\section{The Psychopharmacologists III}

Interviews by David Healy. London: Arnold. 2000. 580 pp. $€ 65.00$ (hb).

ISBN 0340761105

It has been said that scientific progress is made by standing on the shoulders of giants. David Healy must have had a fascinating adventure interviewing the giants that forged psychopharmacology. This is the third volume of his interviews, which have included over 78 contributors, all great or well-known names such as Axelrod and Carlsson (Volume I), Janssen and Schou (Volume II), and Schildkraut, Snyder and Sulser in the present volume.

But did some giants have feet of clay? How is it that the pharmaceutical industry has come to dominate the field? Healy points out that drug companies "are now not simply confined to finding drugs for diseases. They have the power to all but find diseases to suit the drugs they have." Pierre Simon (Sanofi Pharmaceuticals) remarks: "In the 
beginning the pharmaceuticals industry was run by chemists. This was not so bad . . Now most of them are run by people with MBAs ... people who could be the chief executive of Renault, Volvo or anything. They don't know anything about drugs". The problem comes when a chemist presents an interesting drug to the financial analyst, who asks: "What is the market?" The chemist has to decide for what indication the drug will be developed. If the indication is not there, it must be created.

One of many examples of this process was the development in the 1970s of alprazolam (Xanax) for panic disorder. According to David Sheehan (Institute for Research and Psychiatry, Tampa, Florida), the marketing of this drug involved a "clear strategy" to take advantage of the medical profession's confusion in the classification of anxiety disorders; "to create a perception that the drug had special and unique properties that would help it capture market share and displace diazepam from the top position ... There was in fact nothing unique in this regard about Xanax ... benzodiazepines were all good for panic disorder". Xanax was marketed by Upjohn with Food and Drug Administration approval of doses up to $6 \mathrm{mg}$ daily (equivalent to $60-120 \mathrm{mg}$ diazepam). It is perhaps no coincidence, as Healy observes, that the effective incidence of panic disorders has grown a thousand-fold since 1980 .

Sheehan relates a similar 'deliberate tactic' used to market $5-\mathrm{HT}_{1 \mathrm{~A}}$ agonists by generating hysteria in the medical profession about the dangers of benzodiazepines. The $5-\mathrm{HT}_{1 \mathrm{~A}}$ agonists were not a great success, but were succeeded by the selective serotonin reuptake inhibitors (SSRIs), which seemed to show activity in a range of anxiety disorders. Interestingly, according to Healy, the effective incidence of depression, obsessive-compulsive disorder, social phobia, and post-traumatic stress disorder has also increased a thousand-fold since 1980. There are now a number of 'me too' SSRIs because they are cheaper to make than new drugs. Fridolin Sulser of Vanderbilt University notes: "If you can get $20 \%$ of fluoxetine's market, you can make 400-500 million dollars a year with very little investment in research and development". He adds, sadly: "I don't know how to solve this dilemma in an industrial society that is so heavily driven by profits."

Other sinister tales about the machinations of drug companies abound. Ian Oswald describes his well-known battle to get his paper about Upjohn's drug triazolam (Halcion) published, only to find that the then editor of Archives of General Psychiatry was funded by Upjohn and had sent the paper to Upjohn to referee. Pierre Simon relates that reports of adverse effects of sulpiride were censored by the French drug company Delagrange. Raymond Battegay, former professor at the Basel Department of Psychiatry, tells of withdrawal of support by Roche of a study comparing moclobemide with cognitive-behavioural psychotherapy: "apparently they feared that psychotherapy would have the same effect as the drug".

And yet, this book is not a diatribe against the pharmaceutical industry. It must be admitted that extensive clinical trials are too expensive to carry out without drug company support. We all depend on the industry to bring money into academia. A study of a drug that reduces platelet aggregation involved 500 researchers and 20000 patients tested for 3 years. It cost Sanofi around \$250 million. No academic or clinical department can compete with Sanofi's budget for research, which, Simon remarks, is larger than that of the whole French INSERM. Although drug companies pay little attention to brain mechanisms, and theory is secondary to the development of drugs, it must also be admitted that much scientific progress has resulted from the use of drugs such as chlorpromazine, imipramine, benzodiazepines and fluoxetine.

Thus, it seems that money, not science, drives pharmacology. What can the medical profession do? A word of advice about training is given by Solomon Snyder of Johns Hopkins University, Maryland: "It is important . . . to come up with new ideas. PhDs are very well trained to be so critical that they criticise their own thoughts, so they're never creative . . . People without such fancy training . . . don't know about that and just try to think new thoughts. My own psychology is if somebody said day is day, I might say maybe day is night, and that attitude always helps". Further advice from other contributors is to stick close to the bedside: it is salutary to note that the novel effects of chlorpromazine and of imipramine were first observed by nurses. The discovery of these and many other drugs was serendipitous of which Simon offers a definition: "A young man looking for a pin in a haystack finds the farmer's daughter... . if the guy is not interested in the farmer's daughter, he has no chance of doing anything". Perhaps the message is that academic psychopharmacologists should search for the farmer's daughter instead of the pin, but get the drug companies to pay for her dowry.

Readers of this book are advised to peruse it piecemeal. To enjoy a stroll in the company of giants, it is best to walk with them one at a time. Those who do so will find many thought-provoking ideas and may begin to question the widespread assumption that there is a drug treatment for every psychiatric illness, whether already recognised or yet to be described. As Professor Battegay disarmingly remarks to his interviewer: "I don't know, Dr. Healy, if psychiatry is really necessary ....".

Heather Ashton Emeritus Professor of Clinical Psychopharmacology, Department of Psychiatry, Royal Victoria Infirmary, Queen Victoria Road, Newcastle uponTyne NEI 4LP, UK

\section{Psychiatric Management in Neurological Disease}

Edited by Edward C. Lauterbach. Washington, DC: American Psychiatric Press. 2000. 346 pp. $€ 31.50$ (hb). ISBN 0880487860

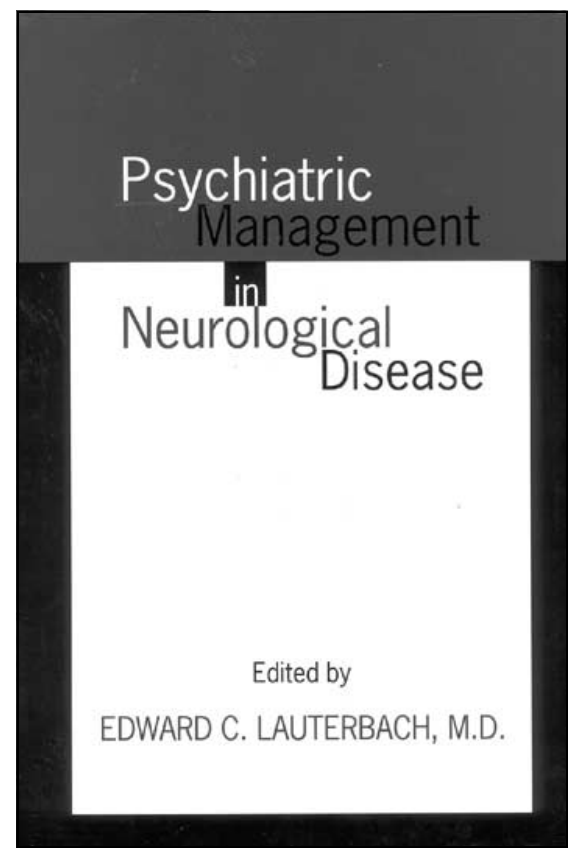

The presence of a psychiatrist on a neurological ward is, of course, no longer novel, but it remains an occurrence that is too infrequent for the full and proper management of many patients with neurological 